\title{
Self-referencing techniques in photonics sensors and multiplexing
}

\author{
Carmen Vázquez, Julio Montalvo, David S. Montero, Pedro C. Lallana \\ Displays and Photonics Applications Group, Dpto. Tecnología Electrónica, Escuela Politécnica \\ Superior, Carlos III University of Madrid, Avda. Universidad 30, 28911 Leganés, Madrid, Spain
}

\begin{abstract}
A short review of self-reference techniques for remote fiber-optic intensity sensors and possible integration in multiplexing sensor networks is reported. Special focus is given to developments on radio-frequency (RF) source modulation techniques in interferometric configurations operating under incoherent regime. Experimental results on ring resonator (RR) configurations in transmission and reflection modes are included. Sensitivity, optimum insertion losses and robustness to intensity error fluctuations are reported. Sensors are interrogated at two sub carrier frequencies having a high rejection of interference from laser source intensity fluctuations and loss in the fiber lead. Dependence on source coherence is also analysed.

Scalable self-referencing sensor networks with low insertion losses implemented in Coarse Wavelength Division Multiplexing (CWDM) technology are reported. The possibility of remote self-referenced measurements using a fullduplex fiber down-lead tenths of kilometers long with no need for optical amplification is also described. Fiber Bragg gratings (FBG) are used in the reflection configuration, thus increasing the sensitivity of the optical transducers. Lowcost off-the-shelf devices in CWDM and DWDM technology can be used to implement and scale the network.
\end{abstract}

Applications to specific photonic sensors are also envisaged and these techniques can be used in networks of microfiber loop resonators, being the microfiber loop the sensing element itself.

Keywords: remote sensing, self-referencing, fiber-optic sensor, ring resonator, micro-fiber loops

\section{INTRODUCTION}

Intensity based sensors are inherently less sensitive than their interferometric counterparts as they rely on amplitude instead of optical-phase. In addition, the transducing mechanism used for converting physical or chemical measurands into a modulation of light intensity sometimes shows poor linearity. However, these limitations do not prevent intensity modulated sensors from having a large potential. They still benefit from the intrinsic advantages of optical detection such as remote sensing, electromagnetic immunity and security in hazardous environment; they can use available standard fibers and sources; the transducers are simple and can easily be made selective to specific measurands; no sophisticated detection system is needed, absolute measurements with high bandwidth can be taken and multiplexing of a number of sensors on the same fiber lead can be made. Because of that there is a wide variety of intrinsically safe photonics sensors in which the external parameter of interest modulates the attenuation of an optical signal [1].

A serious drawback of loss modulation is interference from variation in loss due to long term aging of optical source as long as short-term fluctuations of optical power loss in the fiber lead used to operate the remote sensor, which can occur for example in fiber bends. It is needed a reference signal for calibration out of the sensor response, which undergoes all other losses in the system, and which is multiplexed in the same fiber lead as the measurement signal. Spatial referencing is the simplest to implement with the reference and measurand signals being located within two separated optical fibers [2]; but it is difficult to ensure similar common-mode variations in two separated fibers. Time division [3], wavelength normalization [4], spectral splitting [5], frequency-based self-referencing methods on differential amplitude [6-9] or using amplitude to phase conversion [10], and others using counter propagating signals [11] or electronic means [12] have been reported to overcome this problem.

In the frequency-based self-referencing techniques, the input light is RF modulated and an amplitude ratio measurement are performed at the reception [6-9] or intensity modulations are converted into electrical phase modulation [10, 13]. Different interferometric schemes such as Mach-Zehnder, Michelson [7] and RR topologies [6, 8-9] are considered in the

Photonic Materials, Devices, and Applications II, edited by Ali Serpengüzel,Gonçal Badenes, Giancarlo Righini Proc. of SPIE Vol. 6593, 65931X, (2007) · 0277-786X/07/\$18 - doi: 10.1117/12.723743 
amplitude ratio measurements. In the RR topology an optical ring resonator with a fiber-optic sensor (FOS) in the RR cavity is placed at each remote measuring point and two fiber leads are used. Each sensor is interrogated at two RF frequencies, the reference and sensing channels. Reference channel is not dependent on FOS induced loss, just the opposite happens with sensing channel. These techniques can be used in networks of microfiber loop resonators, being the microfiber loop the sensing element itself [14].

Another relevant aspect of remote sensing is the possibility of multiplexing the response of multiple points to get a quasidistributed sensing scheme. So it is very important to be able to conjugate self-referencing and multiplexing [15-16]. This is one of the main reasons for migration to single-mode fiber (SMF) configurations in self-referenced intensity based sensors networks. Wavelength division multiplexing through fiber Bragg gratings (FBG) is the most common technique. On the other hand, sometimes spectral splitting technique developed in a non efficient manner by using costly dedicated devices such as wavelength sensitive couplers has brought the need of amplification. Solutions based on low cost off-the-shelf devices, optimising power loss to increase the fiber lead length and the number of multiplexed sensors has also been reported [17] and it will be described in this paper.

\section{MOTIVATION}

Simple fiber-optic intensity sensors (FOS) modulate the loss of a fiber path to provide the measurement as an optical intensity modulation signal. A serious drawback of loss modulation is happens when optical power variations have a different origin than the magnitude of interest, because of unpredictable changes in losses of passive components such as fiber leads, optical couplers or connectors, which may change in time and because of aging and environmental conditions (see Fig. 1)

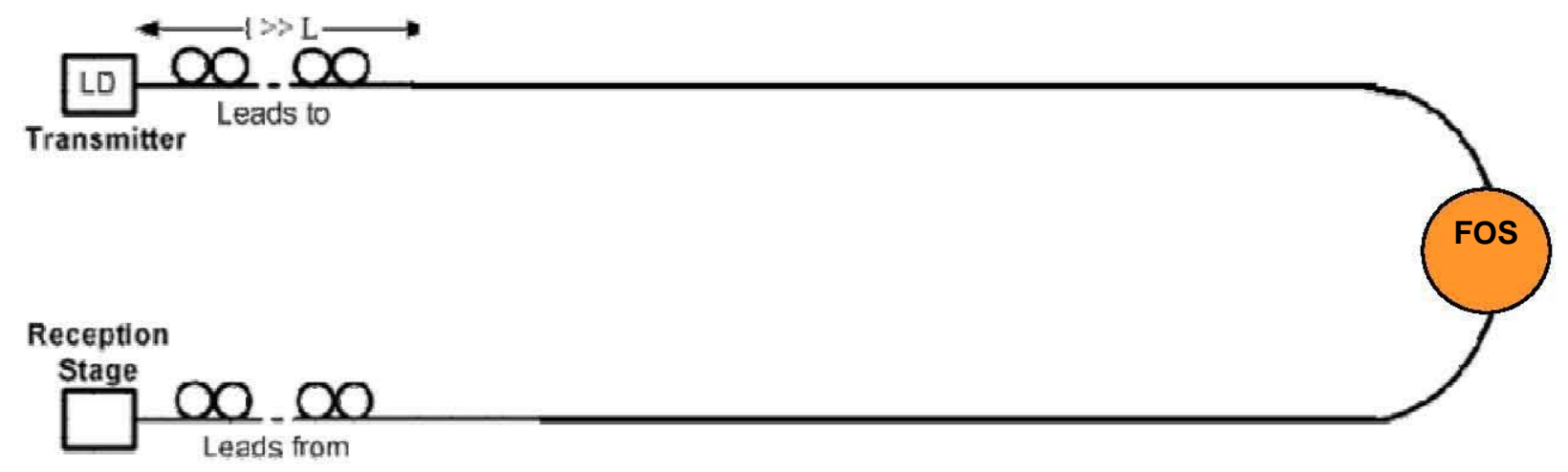

Fig. 1. Motivation for self-reference techniques in FOS remote operation. Arbitrary optical source can be used.

Additionally, random power fluctuations of optical sources at the input of the network and detector sensitivity changes can induce an intensity noise added to the desired signals. In order to neutralize all these effects, a variety of selfreferencing techniques for intensity sensors have been reported [3-11].

\section{RF TECHNIQUES}

There are different techniques based on RF modulation of input signal and amplitude or phase detection at different frequencies to overcome intensity fluctuations modifying measurements accuracy. RF technique operation principle can be seen in Fig. 2, where measurements on RF transfer function of an optical interferometric configuration are reported. FOS loss at the measuring point modifies the frequency response of the interferometric configuration on sensing frequency $f_{1}$ but not on reference channel, $f_{2}$. Both signal $f_{1}$ and $f_{2}$ should travel the same physical path so intensity fluctuations happening outside the interfermetric path will modify both signals in the same quantity, while intensity fluctuations in the interferometric path will modify the transfer function. So the sensing element, FOS, will be located in 
the interferometric path. Measurements on Fig. 2 are taken on a RF spectrum analyser with a tracking generator which modulates a semiconductor LD at 1550nm. Phase differential measurements in a Fabry-Perot topology are reported in [13]. Amplitude differential measurements using a Ring Resonator (RR) with multimode fibers [6], and more recently a Michelson [15] and RR [11] topology have been proposed with SM fibers.

On the following we are going to describe RR based topologies on transmission and reflection configurations.
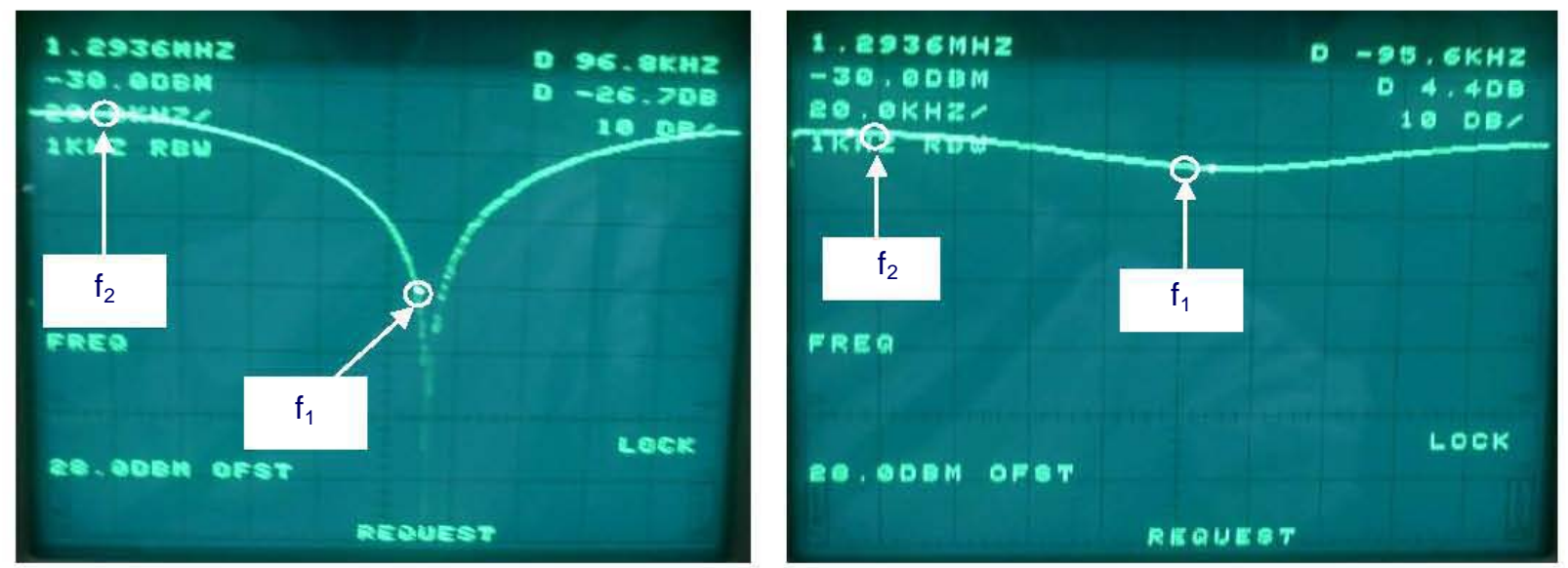

Fig. 2. Principle of operation: $f_{1}$ sensing channel, $f_{2}$ reference channel

\subsection{Simple RR configuration}

Different configurations such as ring resonators operating under incoherent regime have been proposed The RR selfreferencing technique in a transmission configuration $[6,8]$ is made of a RR with an optical fiber sensor, FOS, in the loop which it is placed at each remote measuring point. A scheme of this configuration can be seen in Figure 3.

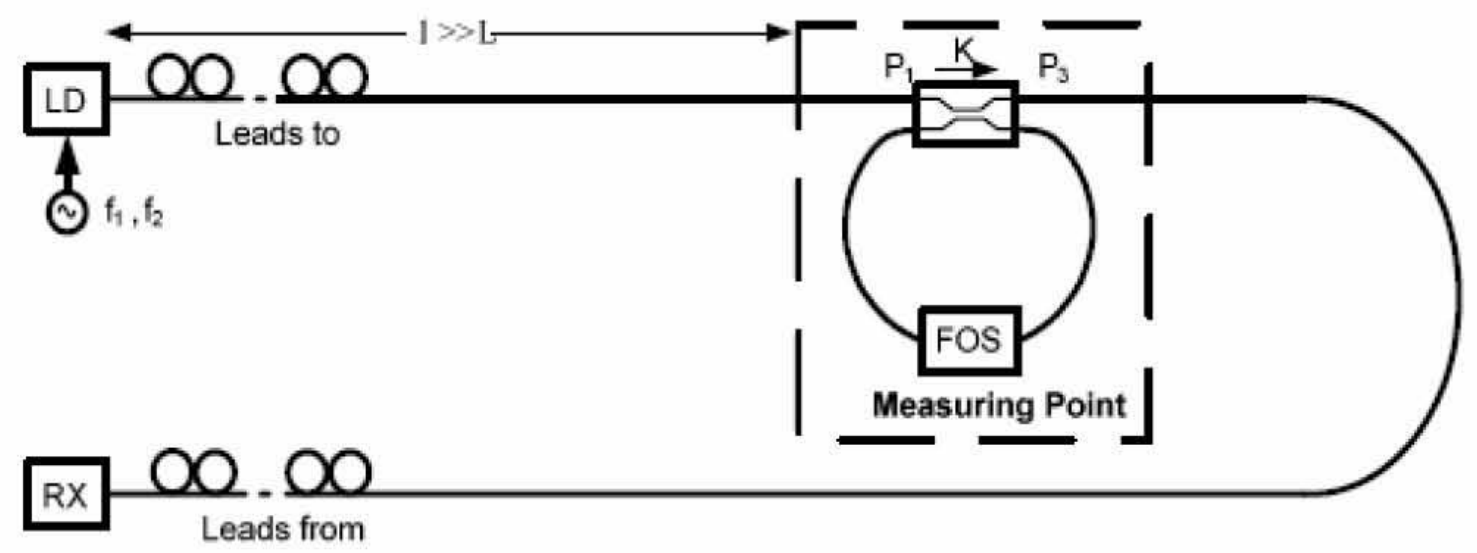

Fig. 3. RF based techniques using ring resonators in transmission configuration: $\mathrm{f}_{1}$ sensing channel, $\mathrm{f}_{2}$ reference channel

The RR configuration can be analyzed in coherent or incoherent regimen, in the following we are going to consider that the optical source coherence length is much lower than loop length $L$, so incoherent optical interference regime is performed. 
Equations describing the behaviour of the device can be derived using $\mathrm{z}$ transform techniques, considering the RR as a RF optical filter [18].

Being input power $P_{\text {in }}=P_{1}$ and output power $P_{\text {out }}=P_{3}$ (see Fig. 3), the transfer function is given by:

$$
\left|H_{p}(z)\right| \equiv\left|\frac{P_{\text {out }}}{P_{\text {in }}}\right|=\Gamma\left|\frac{z-z_{c}}{z-z_{p}}\right|
$$

where

$$
\begin{gathered}
\Gamma=K \cdot(1-\gamma) \\
Z_{c}=\frac{(2 \cdot K-1) H}{K} \\
Z_{p}=K \cdot H
\end{gathered}
$$

In those equations $K$ and $\gamma$ represent the direct power coupling factor and excess losses of the optical coupler, $L$ is the loop length and the global loop power balance, $H$, is given by:

$$
H=10^{-\alpha \cdot 1 / 10} \cdot F(m) \cdot(1-\gamma)
$$

where $\alpha$ is power losses of optical fiber in $\mathrm{dB} / \mathrm{m}$; and $F(m)$ is the optical fiber sensor calibration versus the external magnitude, $m$.

Assuming that input optical power is constant as a function of normalized pulsation $\Omega=2 \cdot \pi \cdot f \cdot \tau$, being $f$ the modulation RF and $\tau$ the loop transit time; a differential measurement is achieved by modulating optical channels with two radio frequencies $f_{1}, f_{2}$ and using a $\mathrm{RR}$ in transmission configuration at each measuring point; being $f_{2}$ the reference signal for calibration, and $f_{1}$ the sensor signal depending on sensor response $F(m)$ (see Fig. 2) .

The self-referencing parameter, $R_{M, n}$, defined as in [8] for a generic node $n$ is given by:

$$
R_{M, n}(\Omega) \equiv \frac{\left|P_{\text {out }, n} / P_{\text {in }, n}\right|_{z=\exp (j \Omega)}}{\left|P_{\text {out }, n} / P_{\text {in }, n}\right|_{\substack{z=\exp (j \Omega) \\ \Omega=2 \cdot \pi \cdot \cdot \cdot \cdot \cdot \tau}}}=\left|\frac{P_{\text {out }, n}(\Omega)}{P_{\text {out }, n\left(\Omega=2 \cdot \pi \cdot f_{2} \cdot \tau\right)}}\right|
$$

From Eqs. (1) and (3), it is derived that the reference frequency is given by:

$$
f_{2}=\frac{i}{\tau} ; \quad i=0,1,2 \ldots
$$

And the $H$ value for the resonance condition, making $\left|H_{p}(z)=0\right|$, and named $H_{0}$ is given by:

$$
H_{0}=\frac{K}{1-2 \cdot K}
$$

RR relative output power given by Eq. (1) can also be expressed as:

$$
\left|\frac{P_{3}}{P_{1}}\right|=\left[(1-\gamma)^{2} \cdot \frac{K^{2}+[(1-2 \cdot K) \cdot H]^{2}+2 \cdot K \cdot(1-2 \cdot K) \cdot H \cdot \cos (\omega \cdot \tau)}{1+(K \cdot H)^{2}-2 \cdot K \cdot H \cdot \cos (\omega \cdot \tau)}\right]^{1 / 2}
$$


From Eq. (9) reference frequency, $f_{2}$ and resonance condition, $H_{o}$ can also be derived.

In terms of sensor linearity, the RR is not a very favorable configuration, unless the measuring range covers a small variation in $H$ [8]. In many cases the FOS itself is not linear, so this should not be considered a constraint.

It is important to determine the design restrictions of the technique. It is derived from Eq. (8) that the resonance condition, $H_{0}$, is achieved without using gain only for $K<1 / 3$. On the other hand, RR insertion losses, $I L_{R R}$, can be derived using Parseval's identity:

$$
I L_{R R}=\frac{1}{N} \cdot \sum_{i=0}^{N-1}\left|H\left(z=\exp \left(\frac{j 2 \cdot \pi \cdot i}{N}\right)\right)\right|^{2}, \quad N \rightarrow \infty
$$

$I L_{R R}$ is $4 \mathrm{~dB}$ for $K=1 / 3$ being $H$ around the resonance condition, $H=H_{0}$, and increasing up to $15 \mathrm{~dB}$ for $\mathrm{K}=0.99$.

\subsection{Ring resonator reflection configuration}

A reflection configuration is made by adding a fiber Bragg grating (FBG) at the RR output fiber in the transmission configuration (see Fig. 4).

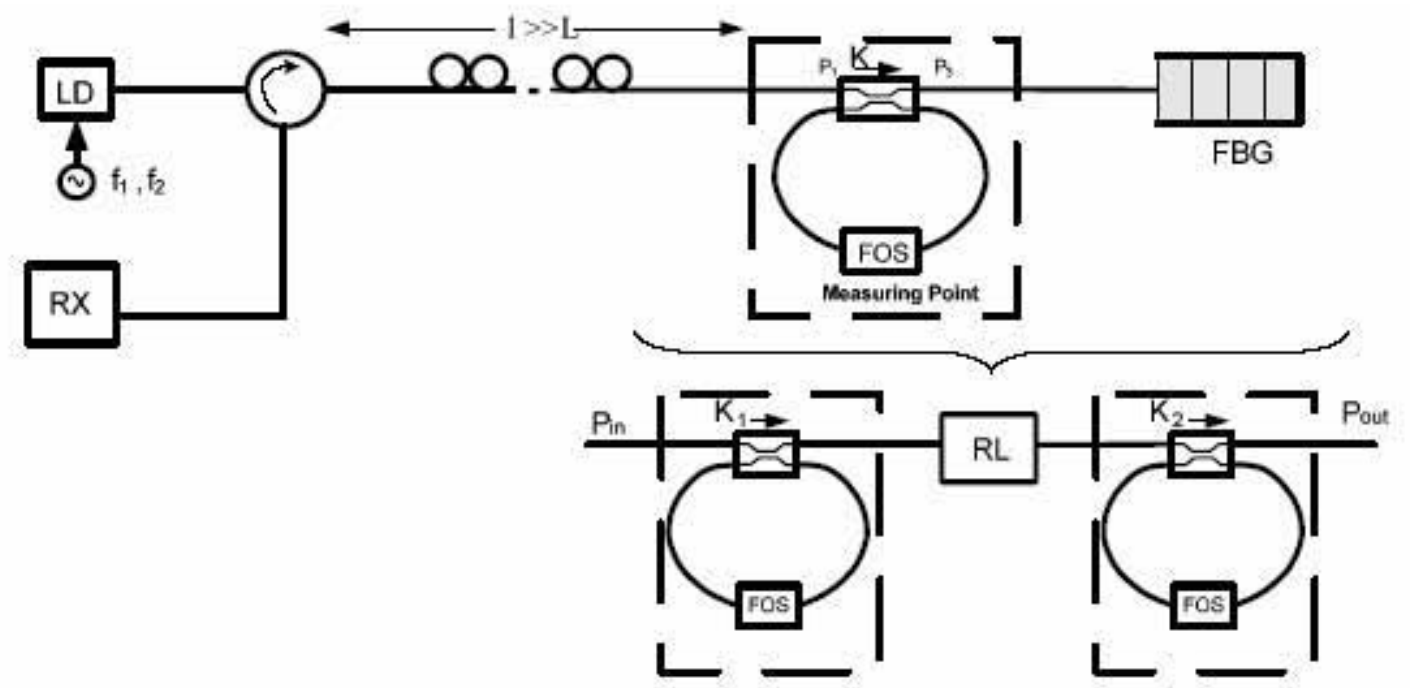

Fig. 4. RF based techniques using ring resonators in reflection configuration: $\mathrm{f}_{1}$ sensing channel, $\mathrm{f}_{2}$ reference channel

For analyzing the reflection configuration behavior it is going to be used the symmetric decomposition shown in Fig. 4, two RR in series and assuming bidirectional intensity sensor response.

$P_{\text {in }}=P^{+}$is the forward input optical power at port 1 and $P_{\text {out }}=P_{1}^{-}$is the backward output optical power at port 1 . RL represents the fiber Bragg grating reflection losses. This configuration optimizes the second-order filter response in terms of symmetry, because the two rings are physically the same, and the same point of operation for the two equivalent resonators can be achieved with one sole adjusting process as regards coupling factor $\left(K_{1}=K_{2}\right)$ and loop length $\left(L_{1}=L_{2}\right)$. Because of that, the configuration is insensitive to coupling factor and loop length fabrication tolerances for symmetric bidirectional optical couplers and intensity sensors. The Z-transform magnitude of the power transfer function impulse response is given by:

$$
\left|H_{p}(z)\right| \equiv\left|\frac{P_{\text {out }}}{P_{\text {in }}}\right|=\Gamma\left|\frac{z-z_{c}}{z-z_{p}}\right|^{2}
$$


where

$$
\Gamma=R L \cdot K^{2} \cdot(1-\gamma)^{2}
$$

$z_{c}$ and $z_{p}$ have the same values as those reported in Eq. (3) and Eq.(4) for a transmission configuration. Using the definition given in Eq. (6) for the self-referencing parameter, $R_{M, n}$, and analysing the resultant response, it can be seen that $f_{2}$ and $H_{o}$ values are the same ones as those in the RR transmission configuration described below.

There is a ratio of 2 between theoretical logarithmic responses of RR reflection and transmission configurations, as expected from Eq. (11).

On the other hand, insertion losses double in the RR reflection configuration. A comparison between RR transmission and reflection configuration normalized frequency responses are reported in [9].

\subsection{Measurements and applications}

The RR self-referencing technique in transmission (see Fig. 3) and reflection configuration (see Fig. 4) were implemented with standard single-mode fiber with FC/APC connectors, and FBG with index matching gel at its transmission port. FOS is simulated in the experiments by a variable optical attenuator (VOA). RR loop length is $100 \mathrm{~m}$ (without considering coupler and VOA pigtails), and a fixed optical coupler was used to ensure stable bidirectional operation. Optical source was externally modulated with a RF signal generated inside a Lightwave Component Analyzer (LCA). A circulator was used for extracting the backward signal in the downlead fiber. Two set of measurements were taken, first to validate the theoretical model and secondly to verify the isolation of the measurements from the effects of downlead attenuation and optical source power fluctuations. $K$ values are chosen so that optical amplification is not needed and the RR insertion losses are acceptable.

RR transfer function in transmission and reflection configuration with $K=0.207, H=0.7, \gamma=0.05$, can be seen in [9]. It can be seen a good agreement between theory and measurements and the double sensitivity for the reflection configuration is verified. On validating the self-referencing technique, we have emulated the main fiber lead using another VOA in the downlead, adjusting the attenuation to induce losses from $0 \mathrm{~dB}$ to $8 \mathrm{~dB}$. A set of $R_{M}$ measurements are taken for each downlead attenuation value for a RR reflection configuration with $K=0.207, f_{1}=53.35 \mathrm{MHz}$ and $f_{2}=53.86 \mathrm{MHz}$. Measurements can be seen in [9], having a relative error below $0.6 \%$. System resolution in terms of optical loss in $\mathrm{dB} / \sqrt{\mathrm{Hz}}$ is around $0.002 \mathrm{~dB} / \sqrt{\mathrm{Hz}}$.

\section{(a)}

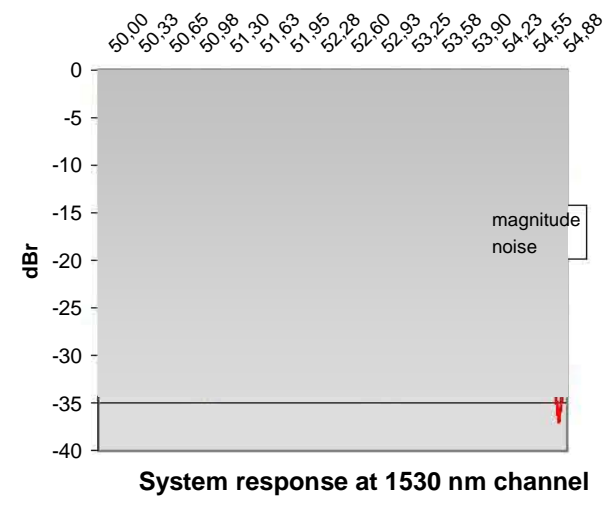

(b)

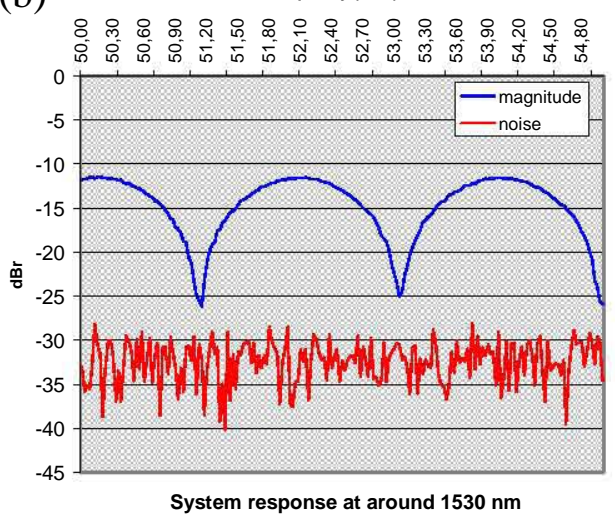

Fig. 5. Magnitude and noise RR transmission configuration transfer function measurements: (a) EDWA source, (b) LD source.

New measurements have also been taken with two different sources, using a LD with a linewidth of $10 \mathrm{MHz}$ and a broadband source, an EDWA of $30 \mathrm{~nm}$ for showing different spectral noise behaviours. As expected theoretically, for a LD source (see Fig. 5) the minimal noise density occurs at the minima of the magnitude transfer function [19], thus partially compensating the RF signal power decrease with regards to signal to noise ratio. Those minima do not appear 
when exciting with the broadband source. As the phase-induced noise does not depend on the RF power at the sensor frequency $f_{2}$, the pre-emphasis technique can be implemented by increasing the modulation index corresponding to that frequency. In situations where the phase noise must be suppressed completely, a differential detection technique could be employed in order to reduce drastically the phase-induced intensity noise [20].

The transmission configuration has been used for referencing a multimode fiber strain sensor [21] and a single-mode optical fiber sucrose evanescent-field sensor with a polymeric nano-film sensitive to humidity [22].

\section{MULTIPLEXING TECHNIQUES}

Scalable multiplexing schemes to poll a large number of passive sensors or sensing regions from a single optoelectronics unit, reducing the number of components will greatly enhance the commercial potential of optical sensing technology. Power budget is one of the key parameters to optimize for increasing the number of sensor in remote operation. There are different sensor multiplexing technologies such as time division multiplexing (TDM), frequency division multiplexing (FDM), wavelength division multiplexing (WDM) among others (see Fig. 6) and network sensors physically connected with different topologies (ladder, star..) and in transmission (two lead fibers) or reflection (a single lead fiber) configurations; a detailed review can be seen in [23]. It is very important to simultaneously design the selfreference technique and the compatible multiplexing technology. Bus and star reflective topologies using FBG have been reported [24-25], employing optical amplification in order to compensate insertion losses of optical couplers; nevertheless, the noise associated to amplification is a limiting aspect. Partial optimizations of power budget using WDM devices at the reception stage have been demonstrated in self-referencing intensity networks [16], but the use of couplers leads to lossy distribution of lightwave channels.

(a)

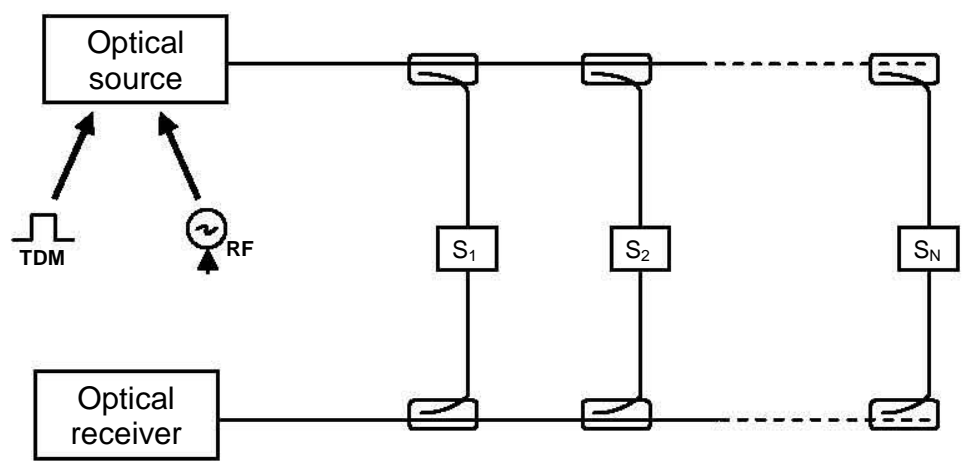

(b)

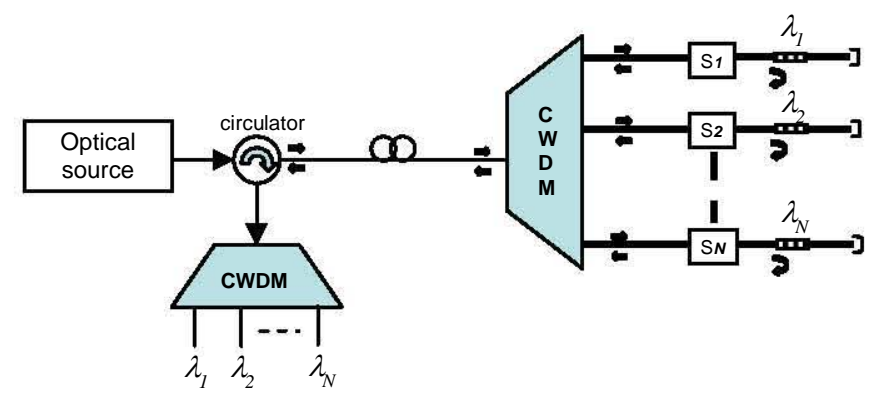

Fig. 6. General self-reference sensors network: (a) Ladder transmission configuration, TDM or FM multiplexing, (b) Star reflective configuration WDM multiplexing. 
A scalable self-referencing sensor network with low insertion losses implemented in Coarse Wavelength Division Multiplexing (CWDM) technology is reported. It allows obtaining remote self-referenced measurements with a fullduplex fiber downlead up to tenths km long, with no need for optical amplification [17]. Fiber Bragg gratings (FBG) are used in order to achieve a reflective configuration, thus increasing the sensitivity of the optical transducers. Low-cost off-the-shelf devices in CWDM technology can be used to implement and scale the network. Ring resonator (RR) based incoherent interferometers at the measuring points are used as self-referencing technique. In the following a theoretical analysis of power budget of the topology is reported, with a comparison between the proposed network and a conventional star topology.

Assuming a generic scalable star topology with a cascade of 2x2 devices (an example can be seen in Fig. 6) to distribute the optical channels to a maximum number of optical sensors $N$, the final ratio between output optical power $\left(P_{R X}\right)$ and input optical power $\left(P_{T X}\right)$ is given by:

$$
\frac{P_{R X}}{P_{T X}}=\left(10^{-2 \alpha \cdot L_{\text {link }} / 10}\right) \cdot\left(R R_{I L}\right)^{2} \cdot I L_{d}^{\log _{2}(N)} \cdot R_{F B G}
$$

In Eq. (13), $P_{T X}$ is the optical power launched into the fiber lead and $P_{R X}$ is the reflected received power; $I L_{d}$ represents the bidirectional insertion losses of the devices used to implement the star, respectively; $\alpha, L_{\text {link }}$ are the attenuation coefficient of the main fiber lead in $\mathrm{dB} / \mathrm{km}$ and the lead length in $\mathrm{km} ; R R_{I L}$ and $R_{F B G}$ are the bidirectional intrinsic insertion losses of the self-referencing configuration and the reflection losses of the FBG respectively.

The number of distribution stages using M-channel CWDM devices is $\log _{M} N$, so the required stages to multiplex $N$ sensors can be reduced by increasing the number of ports of the demultiplexer $(M)$. So Eq. (13) can be used but with this new power of $I L_{d}$. In the case of Fig. 6(b), as $M=N$ the power affecting $I L_{d}$ is the unit.

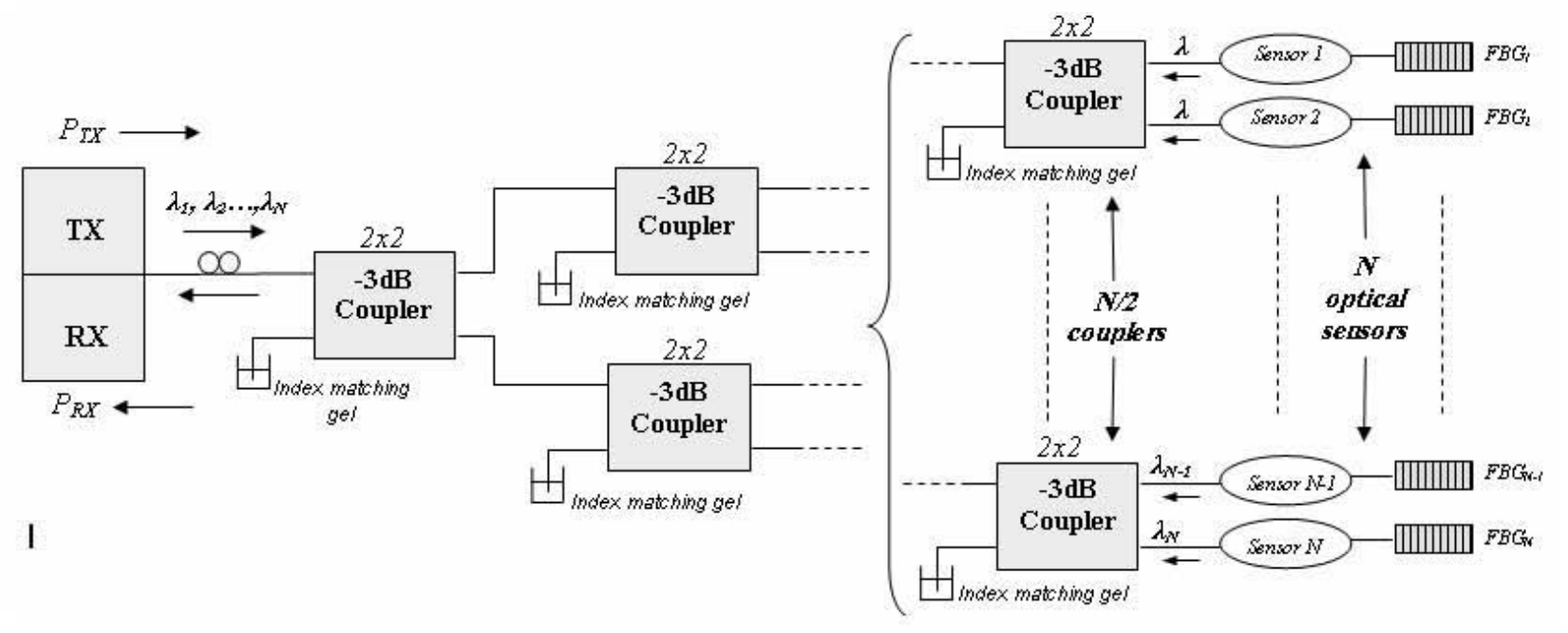

Fig. 7. Passive WDM star topology for intensity sensors using $3 \mathrm{~dB}$ couplers.

The splitter star is established cascading $1 \times 2$ bidirectional power-splitters (see Fig. 6) with $3.15 \mathrm{~dB}$ insertion loss per channel at each direction of light propagation, including polarization-dependent-loss (PDL) and excess loss for commercial couplers. 
The 4 channel CWDM insertion losses are $3.5 \mathrm{~dB}$ per channel and per direction of light propagation including PDL. In the case of the DWDM upgrade of the 4-CWDM star deploying a 40 channel AWG, the total losses per channel are 12 $\mathrm{dB}$ including PDL. The total power loss at each reflective RR-based FOS is $10 \mathrm{~dB}$ per channel.

Using Eq. (13) it can be calculated the maximum values of the main fiber lead length versus the total insertion losses, for the two network schemes under comparison, and for the maximum number of multiplexed optical transducers on the G.694.2 spectral grid and it is shown in Fig. 8. There are two different regions in the graph; the positive length span includes possible implementations of the topologies, while the region of negative distances is not feasible. Therefore, the 4-CWDM addressed sensors could be emplaced around $20 \mathrm{~km}$ further than using the 3-dB couplers power distribution approach.

In the case of a large number of sensors and maintaining the transmitted power per channel, a 40 channel upgrade with DWDM technology, in a single channel of the CWDM network, is still useful; while a 32 channel star with the 3-dB couplers power distribution would be unfeasible, showing around $20 \mathrm{~dB}$ of relative power penalty with regards to the DWDM sensor network.

The CWDM self-reference multiplexing configuration has been experimentally demonstrated in [17]. This topology can also be used for addressing microfiber loop resonators which have been proposed as temperature sensors [14], and for addressing microring resonators on side-polished optical fibers [26]; proposed as biochemical sensing platforms.

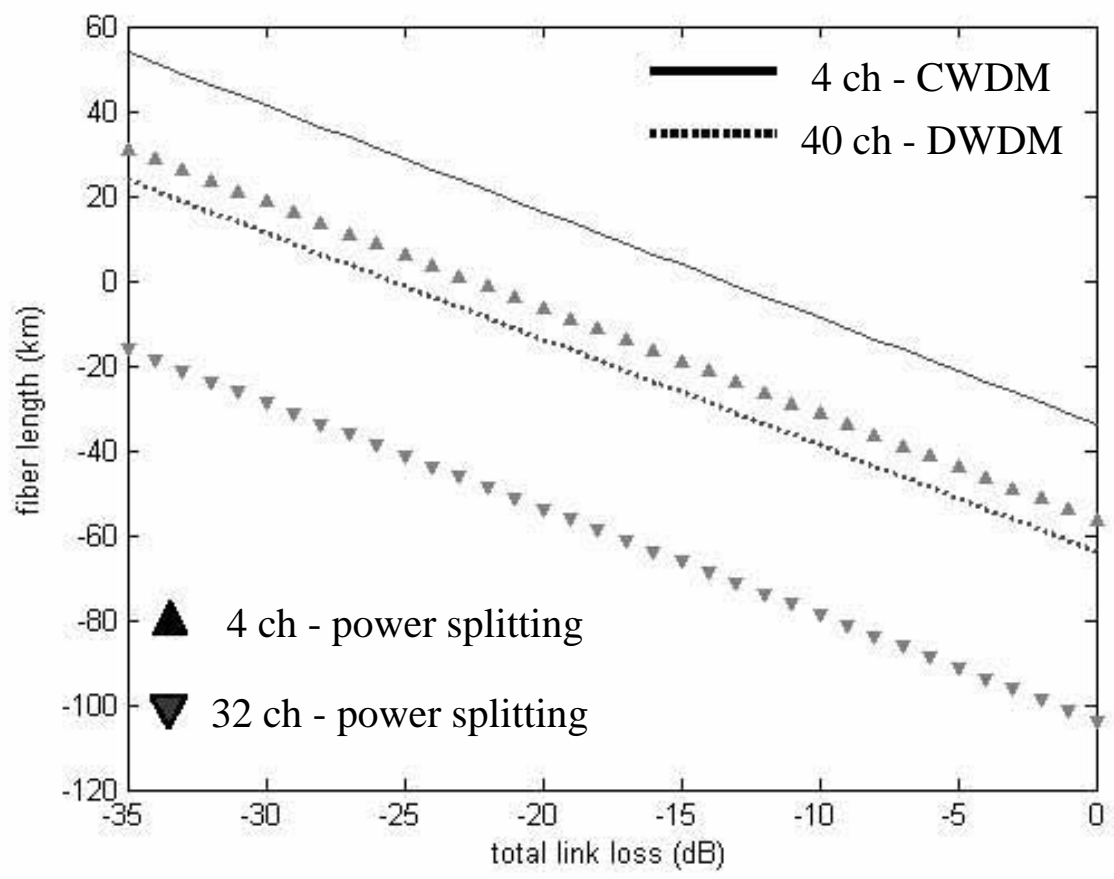

Fig. 8. Maximum fiber length for the reflective star sensor topology in the cases of the WDM (triangles) and the power-splitting (lines) approaches. The feasibility of the network topologies corresponds to fiber lengths $>0 \mathrm{~km}$.

\section{CONCLUSIONS}

A short review of self-reference techniques for remote fiber-optic intensity sensors and possible integration in multiplexing sensor networks is reported. Specific attention is devoted to RF self-referencing techniques based on ring resonator (RR) in transmission and reflection configurations. In the reflection configuration, using fiber Bragg gratings (FBG), the sensor sensitivity doubles and a single down lead fiber is used, but at the expense of doubling the insertion losses. Experimental normalized amplitude and noise frequency responses of RR configurations are reported validating 
the theory. The sensor is interrogated at two RF frequencies (reference and sensor) having a high rejection of interference from optical power fluctuations, relative errors lower than $0.6 \%$ in the measurement parameter are reported in [9]. The sensing concept is particularly favorable in what concerns the minimization of system noise, because what it is monitored is the amplitude of two sinewaves, thus the detection bandwidth can be made as narrow as practically possible, with consequent reduction of the system noise level.

RR based self-reference techniques have been used in MM strain sensors and SM fiber sucrose evanescent-field sensor with polymeric nano-films.

Multiplexing capabilities are also analyzed, by using FBG in a bus or star topology in reflective configurations and/or CWDM multiplexer/demultiplexer in both configurations. By using CWDM networks based on devices with low insertion losses and wavelength dependence, an increase of the fiber lead length and the number of multiplexed sensors for passive star networks can be achieved, while obtaining self-referenced measurements with increased sensitivities. Low-cost off-the-shelf devices can be used to implement the topology and to scale the network with more sensors.

A comparative theoretical analysis considering a passive star topology with $3 \mathrm{~dB}$ couplers has also been presented, concluding that the 4-CWDM addressed sensors could be emplaced around $20 \mathrm{~km}$ further than using the 3-dB couplers power distribution approach.

A real implementation for two multiplexed generic sensors at 1530 and $1550 \mathrm{~nm}$ has been demonstrated, with a fullduplex SM fiber link of $15 \mathrm{~km}$ and a CWDM demultiplexer as optical distribution stage in [17], with $35 \mathrm{~dB}$ crosstalk between optical channels as a consequence of the isolation ratio of the CWDM demultiplexer.

The proposed multiplexing scheme can be applied to any other frequency-based self-referencing mechanism such as

Mach-Zehnder or Michelson configurations. The number of sensors admitted in the new topology can also be scaled easily by replacing one CWDM channel with closer carriers using DWDM technology at the final distribution stages.

\section{ACKNOWLEDGMENTS}

This work was partially supported by CICYT (TEC2006-13273-C03-03-MIC), COST299, EPhoton/One+(NoE-ISTEU), FENIS-CCG06-UC3M/TIC-0619, CAM (FACTOTEM-CM:S-0505/ESP/000417)

\section{REFERENCES}

1. J. W. Berthold, "Historical Review of Microbend Fiber-Optic Sensors", Journal of Lightwave Technology 13(7), 1193-1199 (1995).

2. G. Murtaza and J. M. Senior, "Referenced intensity-based optical fiber sensors." International Journal of Optoelectronics 9(4), 339-348 (1994).

3. W. B. Spillman and J. R. Lord, "Self-referencing Multiplexing Technique for Fiber-Optic Intensity Sensors", Journal of Lightwave Technology 5(7), 865-869 (1987).

4. Masayoshi Kamiya, Hiroaki Ikeda, and Shigenobu Shinohara, "Analog Data Transmission Through Plastic Optical Fiber in Robot With Compensation of Errors Caused by Optical Fiber Bending Loss”, IEEE Transactions On Industrial Electronics 48(5), 1034-1037 (2001).

5. Anbo Wang, Hai Xiao, J. Wang, Zhiyong Wang, W. Zhao, and R. G. May, "Self-Calibrated InterferometricIntensity-Based Optical Fiber Sensors”, Journal of Lightwave Technology 19(10), 1495-1501 (2001).

6. R. I. MacDonald, R. Nychka "Differential Measurement Technique for Fiber-Optic Sensors", Electronics Letters 27(23) 2194-2196 (1991).

7. J. M. Baptista, J. L. Santos and A. S. Lage, "Mach-Zehnder and Michelson topologies for self- referencing fiber optic intensity sensors”, Optical Engineering 39, 1636-1644 (2000).

8. C. Vázquez, J. Montalvo, P.C. Lallana, "Radio-Frequency Ring Resonators for Self-Referencing Fiber-Optic Intensity Sensors”, Optical Engineering Letters 44, 1-2 (2005).

9. C. Vázquez, J. Montalvo, D. S. Montero, J. M. S. Pena, "Self-referencing fiber-optic intensity sensors using Ring Resonators and Fiber Bragg Gratings”, Photonics Technology Letters 18 (22), 2374 - 2376 (2006). 
10. Pierre Sixt, George Kotrotsios, Lucien Falco, And Olivier Parriaux, "Passive Fiber Fabry-Perot Filter for IntensityModulated Sensors Referencing”, Journal Of Lightwave Technology 4(1), 926-932 (1986).

11. C. S. Pérez, A. G. Valenzuela, G. E. S. Romero, J. Villatoro, J. H. Cordero, "Technique for referencing of fiberoptic intensity modulated sensors by use of counterpropagating signals” Optics Letters 29, 1467-1469 (2004).

12. Chang-Da Tsai, Hui-Hsun Huang, Shyh-Lin Tsao, Hsiao-Lung Chan and Chien-Ping Wu, "Error Reduction Of Referenced Intensity-Based Optical Fiber Sensor By Adaptive Noise Canceller”, Electronics Letters 33(1), 982-983 (1997).

13. S. Abad, F.M. Araújo, L.A. Ferreira, J.L. Santos and M. López-Amo, "Interrogation of Wavelength Multiplexed Fiber Bragg Gratings Using Spectral Filtering and Amplitude-to-Phase Optical Conversion”, Journal of Lightwave Technology 21(1), 127-131 (2003).

14. M. Sumetsky, Y. Dulashko, J. M. Fini, A. Hale, and D. J. DiGiovanni, "The Microfiber Loop Resonator: Theory, Experiment, and Application” Journal of Lighwave. Tecnology 24(1), 242-250 (2006).

15. J. M. Baptista, S. Abad, G. M. Rego, L. A. Ferreira, F. M. Araujo and J. L. Santos, "Wavelength multiplexing of frequency-based self-referenced fiber optic intensity sensors”, Optical Engineering 43, 702-707 (2004).

16. S. Abad, M. López-Amo, F. M. Araújo, L. A. Ferreira, and J. L. Santos, "Fiber Bragg grating-based self-referencing technique for wavelength multiplexed intensity sensors,” Optics Letters 27, 222-224 (2002).

17. J. Montalvo, C. Vázquez, D. S. Montero "CWDM self-referencing sensor network based on ring resonators in reflective configuration” Optics Express, 14(11), 4601-4610 (2006).

18. M.C. Vázquez., B.Vizoso, M.Lopez-Amo and M.A.Muriel, "Single and double amplified recirculating delay lines as fiber-optic filters", Electronics Letters 28(11), 1017-1019 (1992).

19. Moshe Tur, Ehud Shafir and Kjell Bløtekjaer, "Source-Induced Noise in Optical Systems Driven by Low-Coherence Sources”, Journal of Lightwave Technology 8, 183-189 (1990).

20. Erwin H. W. Chan and Robert A. Minasian, "Suppression of Phase-Induced Intensity Noise in Optical Delay-Line Signal Processors Using a Differential-Detection Technique”, IEEE Transactions on Microwave Theory and Techniques 54, 873-879 (2006).

21. J. Montalvo, P.C. Lallana, C. Vázquez, "Self-Referencing Fiber-Optic Intensity Strain Sensors”, Optical Fiber Sensors Conference OFS-17, Vol. 5855 (2), 767-770, Brujas , (2005).

22. Jesús M. Corres, Francisco J. Arregui and Ignacio R. Matías, "Design of Humidity Sensors Based on Tapered Optical Fibers”, Journal of Lightwave Technology 22(11), 4329-4336 (2006).

23. J P Dakin, "Multiplexed and distributed optical fiber sensor systems", J. Phys. E: Sci. Instrum.. 20, 954-967 (1987).

24. S. Diaz, M. López-Amo, "Comparison of WDM distributed fiber Raman amplifier networks for sensors," Optics Express 14(4), 1401-1407 (2006).

25. S. Abad, M. López-Amo, "Single and double distributed optical amplifier fiber bus networks with wavelengthdivision multiplexing for photonic sensors," Optics Letters 24, 805-807 (1999).

26. Travis Sherwood, A. Cody Young, Jocelyn Takayesu, Alex K. Y. Jen, Larry R. Dalton, and Antao Chen, "Microring Resonators on Side-Polished Optical Fiber”, IEEE Photonics Technology Letters 17(10), 2107-2109 (2005). 